\title{
GLOBAL BIFURCATION ANALYSIS OF AN ADAPTIVE CONTROL SYSTEM $\dagger$
}

\author{
FATHi M.A. SALAM \\ Department of Electrical Engg., Michigan State University, East Lansing, MI 48824 \\ Stephen A. Van Gils \\ Department of Mathematics \& Computer Sci., Free University, Amsterdam, The Netherlands
}

ZHANG ZHI-FEN

Department of Mathematics, Peking University, Beijing, P.R. China

(Submitted by: J.L. Bona)

\begin{abstract}
We present complete bifurcation analysis of a 2-parameter, 2-dimensional quadratic o.d.e. which arises in the study of Model Reference Adaptive Control (MRAC) systems. The 2-dimensional o.d.e. exhibits saddle-node, (subcritical) Hopf, and saddle-loop (a.k.a. homoclinic connection) bifurcations. We have been able to completely describe the bifurcation diagram by identifying all the bifurcation curves. All but the saddle-loop bifurcation curve have been explicitly characterized. The saddle-loop bifurcation curve, however, has been described qualitatively and its end-points as well as its relative position have been identified.
\end{abstract}

1. Introduction. This study concerns the global bifurcation analysis of a 2dimensional quadratic ordinary differential equation (o.d.e.) which arises in the study of Model Reference Adaptive Control (MRAC) systems [11]. Specifically, the 2-dimensional o.d.e. captures the essential dynamics that a simple adaptive control system may generate in the presence of external disturbances. The dynamics of the equation were first characterized via digital computer simulations in [1] and were found to exhibit various types of bifurcations for a specific set of parameters. Due to the limitation of the computational accuracy of computers, it is essential to verify the bifurcations suggested by the (computer) simulations by analytical means. Moreover, analysis may provide explicit characterization of bifurcation curves and may subsequently lead to the construction of the complete global bifurcation diagram of the system analytically. Such analytical studies do provide support to computer simulations and could bring new insights into alternative choices of system parameters in the design stage of MRAC systems.

This paper is based on the conference version which appeared in [11]. It is organized as follows: In Section 1, we briefly describe the formulation in Model Reference Adaptive Control (MRAC) systems, then we specialize it to the prototype MRAC

Received March 1989, in revised August 1990.

†Research was supported by NSF under grant ECS-8702889.

AMS Subject Classifications: 34, 35, 58F13, 65C99. 
system and the essential 2-dimensional related equation. In Section 2, we determine the number of equilibria and sketch the various phase portraits of the system qualitatively. In Section 3, we study the stability of the equilibrium points in detail and explicitly determine the saddle-node bifurcation curve. We then prove the existence of the Hopf bifurcation and the saddle-loop bifurcation, and give an explicit expression for the Hopf bifurcation curve. As for the saddle-loop bifurcation curve, however, we only specify its end-points and give a qualitative description of its relative position to the other curves. In Section 4, we summarize our results in the form of a theorem.

2. The model reference adaptive control and the prototype adaptive system. Model Reference Adaptive Control (MRAC) systems refer to a control structure for systems modeled as linear time-invariant first order differential equations with unknown parameters. The structure has been constructed with the intent that the overall augmented system (i.e., the system and the associated control structure) may still be described via a set of first order linear equations. This set of equations may in the limit (i.e., as parameters update would have converged) be viewed as time-invariant. Consequently, analysis of MRAC systems has traditionally emphasized linear systems analysis and Laplace transform (frequency) methods.

The engineering idea of MRAC is that one chooses a reference model characterized by a known transfer function or a known set of linear differential equations. The reference model is (forced) by a (chosen) reference signal. Then the standard (feedback) control structure, which is constructed about the system (or plant), uses the same reference signal as its input. The control structure is fixed except for parameters to be specified or, more accurately, to be recursively updated. The parameters update is performed based on the error signals between the output of the system (or plant) and the (desired) output of the reference model. It may also use other accessible signals to achieve successful update. This general description, we hope, will become clear from the following succinct specialization to our prototype adaptive system.

We now describe the usual set-up of MRAC systems specialized to a first order plant with an unknown parameter. We delegate further details to Salam [2] and the references therein. The following defines the main components of the adaptive control system.

$$
\begin{aligned}
\text { Model }: & \dot{x}_{m}=a_{m} x_{m}+r, \\
\text { Plant : } & \dot{x}=a x+u+\lambda, \\
\text { Control : } & u=f\left(x_{m}, x, k, r\right)=-k x+r, \\
\text { Adaptation law : } & \dot{k}=g\left(x_{m}, x, k, r\right)=\gamma\left(x-x_{m}\right) x-\sigma k,
\end{aligned}
$$

where $a_{m}<0$ is a chosen parameter, $r$ is a known reference signal, $a$ and $\lambda$ are unknown constants; $\gamma$ and $\sigma$ are control parameters to be chosen by the designer.

In control theory, $a_{m}$ is referred to as the known stable pole (or eigenvalue) of the reference model, $a$ is referred to as the unknown pole (or eigenvalue) of the plant, and $\lambda$ represents an unknown external disturbance acting on the plant. 
We note that the functions $f$ and $g$ are specified by the designer. The choice of $f$ and $g$ are crucial to the formulations of MRAC systems. The choice of $f$ as in (3) is suggested by the fact that both plant and model are linear systems and by the well-known constant (state) feedback in linear system theory. Because one addresses only linear (time-invariant) plants and models, there is no obvious benefit from modifying $f$ in (3).

The choice of (4) is a consequence of using a quadratic Liapunov function, then guaranteeing that its usual time-derivative along trajectories is less than or equal to zero (see [2] and the references therein).

Now combining (2) and (3), one obtains the representation of the plant augmented with the control; i.e.,

$$
\dot{x}=(a-k) x+r+\lambda .
$$

Equation (5) matches equation (1) provided that we choose $k^{*}$ such that $a_{m}=$ $\left(a-k^{*}\right)$. Note that this matching equality of parameters is facilitated by choosing the control in (3) as linear in $x$.

As usual, one derives the error equations as follows. One defines the output (in this case, state) error as $e=x-x_{m}$. Then one obtains

$$
\dot{e}=\dot{x}-\dot{x}_{m}=a e-k e+\lambda+\left(-a_{m}+a-k\right) x_{m},
$$

where $k$ has its dynamics defined by the adaptation law (4), which now reads

$$
\dot{k}=\gamma e^{2}-\sigma k+\gamma e x_{m} .
$$

Equations (6) and equation (1) describe the dynamics of the overall (augmented) system. Note that the dynamics of the state of the reference model $x_{m}$ (as specified by (1)) is decoupled from the other two states, namely, $e$ and $k$. Note also that equations (6) are forced by the (independent) reference model state $x_{m}$.

Now suppose, for simplicity, that the reference signal $r=0 . \quad(r=0$ is the case referred to in control theory as the regulation problem and it is of interest in its own right $[1,2]$.) Then, the initial condition $x_{m}(0)$ only affects the transient behavior of equations (6); specifically, the steady state solutions are not changed. The qualitative dynamics of the overall system are captured by the dynamics on the plane defined by $x_{m}=0$. We may thus choose the initial condition $x_{m}(0)=0$ so that $x_{m}(t)=0$ for all $t$. Consequently, the dynamics of the adaptive system are captured by the simpler 2-dimensional system

$$
\begin{aligned}
& \dot{e}=a e-k e+\lambda, \\
& \dot{k}=\gamma e^{2}-\sigma k .
\end{aligned}
$$

Once the analysis of equations (7) are completed, the results can be extended to the case when the reference input $r$ is a sufficiently small periodic function [12].

3. The equilibria and the phase portraits. To simplify the analysis, we first rescale system (7). Assuming that $a$ and $\lambda$ are nonzero, we define the transformations

$$
\begin{aligned}
& x=\frac{a}{\lambda} e, \quad y=\frac{1}{a} k, \quad t^{\prime}=a t, \\
& \mu=\frac{\lambda^{2}}{a^{4}} \gamma, \quad \nu=-\frac{1}{a} \sigma .
\end{aligned}
$$


We then get

$$
\begin{aligned}
& \dot{x}=x-x y+1=: F(x, y), \\
& \dot{y}=\mu x^{2}+\nu y=: G(x, y),
\end{aligned}
$$

where the time derivatives are now with respect to $t^{\prime}$. For convenience, however, we shall again use $t$ to denote the time. Observe that system (9) has only two independent parameters $\nu$ and $\mu$.

We divide the parameter space $(\nu, \mu)$ by the lines $\nu=0, \mu=0$ and $\frac{\mu}{\nu}=-\frac{4}{27}$ into six regions as in Figure 1. The singular (equilibrium) points of system (9) are the intersections of the curves $y=1+\frac{1}{x}$ and $y=-\frac{\mu}{\nu} x^{2}$. Within each region and on each boundary, the number of singular points does not change. The $x$-coordinate of the singular points are the roots of the polynomial $\frac{\mu}{\nu} x^{3}+x+1=0$. Note that for a fixed ratio $\frac{\mu}{\nu}$, the roots of this polynomial are fixed. Correspondingly the position (and number) of the singular points of system (9) will be fixed. When $\frac{\mu}{\nu}=-\frac{4}{27}$, the polynomial has double roots $x=-\frac{3}{2}$ which give rise to the degenerate singular point $(x, y)=\left(-\frac{3}{2}, \frac{1}{3}\right)$.

Corresponding to each region and to each boundary in the parameter space $(\nu, \mu)$, the two curves $y=1+\frac{1}{x}$ and $y=-\frac{\mu}{\nu} x^{2}$ divide the whole phase plane $(x, y)$ into several regions. In each of these regions the signs of $\dot{x}$ and $\dot{y}$ are fixed. Consequently, one can sketch the trajectories in the whole $(x, y)$ plane; see [3] and [4]. We have done so and the results are depicted in the various labeled cases of Figures 1 and 2. However, one can not ascertain the existence or otherwise of degeneracies, equilibria at infinity, periodic orbits, and/ or homoclinic orbits.

The sketched phase portraits in Figure 1 depict singular points labeled as $P_{1}$, $P_{2}$, and $P_{3}$, respectively. $P_{1}$ is the label for the singular point residing in the first quadrant of the state space. Observe that its $y$-coordinate is always greater than 1 and its $x$-coordinate is always positive. $P_{2}$ and $P_{3}$ are the labels for the singular points residing in the second quadrant with their $y$-coordinates always less than 1 and their $x$-coordinates always negative. The $y$-coordinate of $P_{2}$ is always $\leq \frac{1}{3}$ and the $y$-coordinate of $P_{3}$ is always $\geq \frac{1}{3}$ (but is $\leq 1$ ).

\section{The complete global analysis.}

4.1. On the nonexistence of periodic orbits surrounding the singular point $P_{2}$ of case $1^{(1)}$ and the singular point $P_{1}$ of cases $7,8,9$. Case $1^{(1)}$ : From case $1^{(1)}$ in Figure $1, \dot{y}>0$ over all the region $y>0$ in the (phase) plane. Also, $\dot{x}>0$ over all the region $x>0, y<0$ in the (phase) plane. Consequently, there is no possibility of a periodic orbit existing in either one of the two regions. This conclusion extends to the (region) complement of $x<0, y<0$. Hence, if (9) has a periodic orbit, it must be entirely located within the simply connected region $x<0, y<0$. However, the divergence of system (9); i.e., $\operatorname{div}(9)=1-y+\nu>0$, is positive (and does not change sign) over any sub-region of region $x<0, y<0$. Consequently, by the Bendixson theorem [4], no periodic orbits are possible in this region either. Thus no periodic orbits are possible in this case. 
Cases 7, 8, 9: Let $H(x, y)=x^{-1}$ be the Dulac function; then the quantity

$$
\begin{aligned}
\frac{\partial(F H)}{\partial x}+\frac{\partial(G H)}{\partial y} & =\frac{\partial F}{\partial x} H+F \frac{\partial H}{\partial x}+\frac{\partial G}{\partial y} H+G \frac{\partial H}{\partial y} \\
& =(1-y) x^{-1}-(x-x y+1) x^{-2}+\nu x^{-1} \\
& =\nu x^{-1}-x^{-2}<0
\end{aligned}
$$

when $x>0$. Hence by Dulac's Theorem [4], there is no periodic orbit surrounding the focus $P_{1}$ in the simply connected region $x>0$.

In all cases, except cases 7 and 8, the qualitative structure of (9) has been completely determined as depicted in the corresponding cases in Figures 1 and 2.

Next we address case 7 , then case 8 .

4.2. Singular points at infinity [4], [5], [6]. In order to determine the stability property of the singular point $P_{3}$ and in order to determine whether the stable and unstable manifolds of the saddle point $P_{2}$ produce a saddle-loop, we transform system (9) to be defined on the Poincare sphere.

Applying the Poincare transformation $x=\frac{1}{z}, y=\frac{u}{z}$ to (9) we get

$$
\begin{aligned}
& \frac{d u}{d t}=-u z F\left(\frac{1}{z}, \frac{u}{z}\right)+z G\left(\frac{1}{z}, \frac{u}{z}\right)=\frac{\mu+(\nu-1) u z+u^{2}-u z^{2}}{z}, \\
& \frac{d z}{d t}=-z^{2} F\left(\frac{1}{z}, \frac{u}{z}\right)=\frac{u z-z^{2}-z^{3}}{z} .
\end{aligned}
$$

Let $z d t^{\prime}=d t$; then we obtain

$$
\begin{aligned}
& \frac{d u}{d t^{\prime}}=\mu+(\nu-1) u z+u^{2}-u z^{2} \\
& \frac{d z}{d t^{\prime}}=u z-z^{2}-z^{3}
\end{aligned}
$$

Singular points of (11) on the line $z=0$ correspond to singular points at infinity of system (9). Since in case 7 the parameter $\mu>0$, system (11) has no singular points on $z=0$.

Now we are going to study the singular point at infinity in the direction parallel to the $y$-axis. Applying the different Poincare transformation $x=\frac{\nu}{z}, y=\frac{1}{z}$ to system (9) we get

$$
\begin{aligned}
& \frac{d \nu}{d t}=z P\left(\frac{\nu}{z}, \frac{1}{z}\right)-z \nu G\left(\frac{\nu}{z}, \frac{1}{z}\right)=\frac{-\nu+(1-\nu) \nu z+z^{2}-\mu \nu}{z} \\
& \frac{d z}{d t}=-z^{2} G\left(\frac{\nu}{z}, \frac{1}{z}\right)=\frac{-\nu z^{2}-\mu z \nu^{2}}{z}
\end{aligned}
$$

Let $z d t^{\prime}=d t$; then we obtain

$$
\begin{aligned}
& \frac{d \nu}{d t^{\prime}}=-\nu+(1-\nu) \nu z+z^{2}-\mu \nu^{3} \\
& \frac{d z}{d t^{\prime}}=-\nu z^{2}-\mu z \nu^{2}
\end{aligned}
$$


Now let $z=0$; then the right hand side of equation (13i) gives $-\nu-\mu \nu^{3}=$ $-\nu\left(1+\mu \nu^{2}\right)$. Since $\mu>0$, we only have the singular point $z=0, \nu=0$.

We are now going to determine the stability property of the singular point $(0,0)$ of system (13).

Let $t^{\prime} \rightarrow-t^{\prime} ;$ then (13) becomes

$$
\begin{aligned}
& \frac{d z}{d t^{\prime}}=F_{2}(z, \nu)=\nu z^{2}+\mu z \nu^{2}, \\
& \frac{d \nu}{d t^{\prime}}=\nu+G_{2}(z, \nu)=\nu-(1-\nu) \nu z-z^{2}+\mu \nu^{3} .
\end{aligned}
$$

Setting $\nu+G_{2}(z, \nu)=0$, we obtain the following analytic expansion of $\nu$ in $z$ : $\nu=z^{2}+a z^{3}+\cdots$, which when inserted into $F_{2}(z, \nu)$ gives $+\nu z^{2}+\mu z^{5}+\cdots$. So the singular point $(0,0)$ is a saddle-node $[4,6]$. Observing that the denominator of system (12) is odd and that we have used the transformation $t^{\prime} \rightarrow-t^{\prime}$, the point $(0,0)$ gives rise to the phase portrait depicted in Figure 3.

Now we are going to prove that the stable manifold of $P_{2}$ in region $y>0$ can not tend to $\infty$ in a direction parallel to the $y$-axis. In region $\dot{x}>0, \dot{y}<0$, the stable manifold can be expressed as a function, say $y(x)$. If $\lim _{x \rightarrow x_{L}} y(x)=\infty$, where $x_{L} \leq x_{P_{2}}<0$, then we will have

$$
\infty=\lim _{x \rightarrow x_{L}} \frac{\mu \frac{x^{2}}{y(x)}+\nu}{\frac{x}{y(x)}-x+\frac{1}{y(x)}}=-\frac{\nu}{x_{L}} .
$$

Thus, the contradiction proves our assertion.

As a consequence, we now have three qualitative possibilities for the stable manifold of $P_{2}$ in region $y>0$. These possibilities are depicted on the Poincare semisphere in Figure 4.

4.3. Hopf bifurcation at $P_{3}\left(x_{0}, y_{0}\right)$. Let the equilibrium point $P_{3}$ have the coordinates $\left(x_{0}, y_{0}\right)$. Let $\bar{x}=x-x_{0}$ and $\bar{y}=y-y_{0}$. System (9) then becomes

$$
\begin{aligned}
& \frac{d x}{d t}=\left(1-y_{0}\right) x-x_{0} y-x y, \\
& \frac{d y}{d t}=2 x_{0} \mu x+\nu y+\mu x^{2},
\end{aligned}
$$

where for simplicity we again write $(x, y)$ instead of $(\bar{x}, \bar{y})$. If Hopf bifurcation occurs, $\left(x_{0}, y_{0}\right)$ must satisfy $(\operatorname{div}(15):=$ divergence of system $(15))$

$$
\operatorname{div}(15)=\left(1-y_{0}\right)-y+\left.\nu\right|_{(0,0)}=1-y_{0}+\nu=0 .
$$

That is, $y_{0}=1+\nu$. And since $y_{0}=1+\frac{1}{x_{0}}$, then $x_{0}=\frac{1}{\nu}$. Now substituting into (15) and letting $t \rightarrow-t$, we get

$$
\begin{aligned}
& \frac{d x}{d t}=\nu x+\frac{1}{\nu} y+x y \\
& \frac{d y}{d t}=-\frac{2 \mu}{\nu} x-\nu y-\mu x^{2}
\end{aligned}
$$


$\frac{2 \mu}{\nu^{2}}-\nu^{2}>0$ is the necessary condition for $(0,0)$ to be a center or a focus of $(16)$. Let $\sigma=\sqrt{\frac{2 \mu}{\nu^{2}}-\nu^{2}}, x=\frac{1}{c} \zeta-\frac{a}{c \sigma} \eta, y=-\frac{1}{\sigma} \eta$, and $t^{\prime}=\sigma t$, where $a=\nu, c=-2 \frac{\mu}{\nu}$. System (16) is now transformed to

$$
\begin{aligned}
& \frac{d \zeta}{d t}=-\eta-\frac{\nu^{3}}{4 \mu \sigma} \zeta^{2}-\frac{1}{\sigma^{2}}\left(1+\frac{\nu^{4}}{2 \mu}\right) \zeta \eta-\frac{\nu}{\sigma^{3}}\left(1+\frac{\nu^{4}}{4 \mu}\right) \eta^{2} \\
& \frac{d \eta}{d t}=\zeta-\frac{\nu^{2}}{4 \mu} \zeta^{2}-\frac{\nu^{3}}{2 \mu \sigma} \zeta \eta-\frac{\nu^{4}}{4 \mu \sigma^{2}} \eta^{2} .
\end{aligned}
$$

Denote

$$
\begin{gathered}
a_{20}=-\frac{\nu^{3}}{4 \mu \sigma}, \quad a_{11}=-\frac{1}{\sigma^{2}}\left(1+\frac{\nu^{4}}{2 \mu}\right), \quad a_{02}=-\frac{\nu}{\sigma^{3}}\left(1+\frac{\nu^{4}}{4 \mu}\right), \\
b_{20}=-\frac{\nu^{2}}{4 \mu}, \quad b_{11}=-\frac{\nu^{3}}{2 \mu \sigma}, \quad b_{02}=-\frac{\nu^{4}}{4 \mu \sigma^{2}} \\
A=a_{20}+a_{02}=-\frac{\nu^{3}}{4 \mu \sigma}-\frac{\nu}{\sigma^{3}}\left(1+\frac{\nu^{4}}{4 \mu}\right) \\
B=b_{20}+b_{02}=-\frac{\nu^{2}}{4 \mu}-\frac{\nu^{4}}{4 \mu \sigma^{2}} \\
\alpha=a_{11}+2 b_{02}=-\frac{1}{\sigma^{2}}-\frac{\nu^{4}}{\mu \sigma^{2}} \\
\beta=b_{11}+2 a_{20}=-\frac{\nu^{3}}{\mu \sigma} .
\end{gathered}
$$

Since $\nu^{8}-4 \mu \nu^{4}<0$, then

$$
\frac{1}{\sigma^{4}}-\frac{\nu^{4}}{4 \mu^{2}}=\nu^{4}\left(\frac{1}{4 \mu^{2}+\nu^{8}-4 \mu \nu^{4}}-\frac{1}{4 \mu^{2}}\right)>0 .
$$

For $\nu<0, \mu>0$ we get

$$
W_{1}=A \alpha-B \beta=\frac{\nu}{\sigma}\left(\frac{5 \nu^{4}}{4 \mu \sigma^{4}}+\frac{\nu^{2}}{4 \mu \sigma^{2}}+\frac{1}{\sigma^{4}}+\frac{\nu^{8}}{4 \mu^{2} \sigma^{4}}-\frac{\nu^{4}}{4 \mu^{2}}\right)<0 .
$$

By the results in [7], and considering the change of the sign of $t$ in (16), $(0,0)$ is an unstable weak focus of order one; it means that under the Hopf bifurcation only one unstable limit cycle will be produced near the singular point $(0,0)$. One may directly use analogous formulae based on the theory of Hopf bifurcation, as was reported in [8].

4.4. On the existence of at most one limit cycle when $1-y_{0}+\nu \leq 0$. We rewrite system (15) in the form

$$
\begin{aligned}
& \frac{d x}{d t}=f_{0}(x)-f_{1}(x) y \\
& \frac{d y}{d t}=g_{0}(x)+g_{1}(x) y
\end{aligned}
$$


where $f_{0}(x)=\left(1-y_{0}\right) x, f_{1}(x)=x_{0}+x, g_{0}(x)=2 x_{0} \mu x+\mu x^{2}$, and $g_{1}(x)=\nu$. Now let $\eta=f_{0}(x)-f_{1}(x) y$. Then (18) becomes

$$
\begin{aligned}
& \frac{d x}{d t}=\eta \\
& \frac{d \eta}{d t}=-\psi_{0}(x)-\psi_{1}(x) \eta-\psi_{2}(x) \eta^{2}
\end{aligned}
$$

where

$$
\begin{aligned}
& \psi_{0}(x)=f_{1}(x) g_{0}(x)+f_{0}(x) g_{1}(x)=\mu x^{3}+3 x_{0} \mu x^{2}+\left(2 x_{0}^{2} \mu+\nu\left(1-y_{0}\right)\right) x, \\
& \psi_{1}(x)=\frac{f_{1}^{\prime}(x)}{f_{1}(x)} f_{0}(x)-f_{0}^{\prime}(x)-g_{1}(x)=\frac{-\left(1-y_{0}+\mu\right) x_{0}-\nu x}{x_{0}+x} \\
& \psi_{2}(x)=-\frac{f_{1}^{\prime}(x)}{f_{1}(x)}=-\frac{1}{x_{0}+x} .
\end{aligned}
$$

Let $\eta=u \exp \left(-\int_{0}^{x} \psi_{2}(z) d z\right)$, then (19) becomes

$$
\begin{aligned}
& \frac{d x}{d t}=u \exp \left(-\int_{0}^{x} \psi_{2}(z) d z\right) \\
& \frac{d u}{d t}=-\psi_{0}(x) \exp \left(\int_{0}^{x} \psi_{2}(z) d z\right)-\psi_{1}(x) u .
\end{aligned}
$$

Let $-\nu=u+\int_{0}^{x} \psi_{1}(s) \exp \left(\int_{0}^{s} \psi_{2}(z) d z\right) d s$, and define the new time transformation

$$
\frac{d t}{d t^{\prime}}=\exp \left(\int_{0}^{x} \psi_{2}(z) d z\right)
$$

Equation (20) gives the Lienard system

$$
\begin{aligned}
& \frac{d x}{d t^{\prime}}=-\nu-F(x) \\
& \frac{d \nu}{d t^{\prime}}=g(x),
\end{aligned}
$$

where

$$
\begin{gathered}
F(x)=\int_{0}^{x} \psi_{1}(z) \exp \left(\int_{0}^{z} \psi_{2}(s) d s\right) d z \\
=\int_{0}^{x} \frac{-\nu z-\left(1-y_{0}+\nu\right) x_{0}}{x_{0}+z} \exp \left(\int_{0}^{z} \frac{-1}{x_{0}+s} d s\right) d z \\
g(x)=x\left(\mu x^{2}+3 x_{0} \mu x+2 x_{0}^{2} \mu+\nu\left(1-y_{0}\right)\right) \exp \left(-2 \int_{0}^{x} \frac{1}{x_{0}+s} d s\right) .
\end{gathered}
$$

Define

$$
a=\frac{-3 x_{0} \mu-\sqrt{x_{0}^{2} \mu^{2}-4 \mu \nu\left(1-y_{0}\right)}}{2 \mu}
$$


and

$$
b=\frac{-3 x_{0} \mu+\sqrt{x_{0}^{2} \mu^{2}-4 \mu \nu\left(1-y_{0}\right)}}{2 \mu} .
$$

Since $-8 x_{0}^{2} \mu^{2}-4 \mu \nu\left(1-y_{0}\right)<0$ is the necessary condition for the eigenvalues of (18) to be complex, this implies that $0<a<b$ and $x g(x)>0$, whenever $x<a$ and $x \neq 0$. So the limit cycles of (18) must be located in the region $x<a$.

Now we are going to prove that the quantity $\left(\frac{f(x)}{g(x)}\right)^{\prime}<0$ whenever $x<a$, where

$$
f(x):=F^{\prime}(x)=\psi_{1}(x) \exp \left(\int_{0}^{x} \psi_{2}(s) d s\right)
$$

Then by a theorem in [9], system (21), and hence (18), has at most one limit cycle. If this limit cycle exists, it will be unstable.

Thus, we calculate

$$
\left(\frac{f(x)}{g(x)}\right)^{\prime}=\frac{\psi_{0}(x) \psi_{1}^{\prime}(x)-\psi_{0}(x) \psi_{1}(x) \psi_{2}(x)-\psi_{1}(x) \psi_{0}^{\prime}(x)}{\psi_{0}^{2}(x)} \exp \left(-\int_{0}^{x} \psi_{2}(s) d s\right)
$$

where

$$
\begin{aligned}
& \psi_{0}(x) \psi_{1}^{\prime}(x)-\psi_{0}(x) \psi_{1}(x) \psi_{2}(x)-\psi_{1}(x) \psi_{0}^{\prime}(x) \\
= & \frac{1}{x_{0}+x}\left\{-\nu x\left[\mu x^{2}+3 x_{0} \mu x+2 x_{0}^{2} \mu+\nu\left(1-y_{0}\right)\right]\right. \\
& \left.+\left[\left(1-y_{0}+\nu\right) x_{0}+\nu x\right]\left[3 \mu x^{2}+6 x_{0} \mu x+2 x_{0}^{2} \mu+\nu\left(1-y_{0}\right)\right]\right\} \\
= & \frac{1}{x_{0}+x}\left[2 \nu \mu x^{3}+3 \mu x_{0}\left(1-y_{0}+2 \nu\right) x^{2}\right. \\
& \left.+6 \mu x_{0}^{2}\left(1-y_{0}+\nu\right) x+x_{0} \nu\left(1-3 y_{0}\right)\left(1-y_{0}+\nu\right)\right] .
\end{aligned}
$$

Since

$$
x_{0}+a=\frac{-x_{0} \mu-\sqrt{x_{0}^{2} \mu^{2}-4 \mu \nu\left(1-y_{0}\right)}}{2 \mu}<0,
$$

then $x_{0}+x<0$, when $x<a$. Limit cycles can only be located in the region $x<c=\min \left(a,-x_{0}-1\right)$; and $\psi_{0}(x) \psi_{1}^{\prime}(x)-\psi_{0}(x) \psi_{1}(x) \psi_{2}(x)-\psi_{1}(x) \psi_{0}^{\prime}(x)<0$, whenever $x<-x_{0}-1$. In the above calculations, we have used the inequalities $-1<\nu<0$ and $0<\mu<-\nu^{3}(1+\nu)$, which will be proved in 4.5. Thus we conclude that $\left(\frac{f(x)}{g(x)}\right)^{\prime}<0$, whenever $x<c$.

4.5. On the existence of the saddle-loop curve $\ell_{3}$ and its relative position to the Hopf bifurcation curve $\ell_{1}: 1-y_{0}+\nu=0$ and the curve $\ell_{2}: 1-y_{0}^{*}+\nu=0$. Let the label $P_{2}\left(x_{0}^{*}, y_{0}^{*}\right)$ denote the equilibrium point $P_{2}$ and its coordinates $\left(x_{0}^{*}, y_{0}^{*}\right)$. Let the label $P_{3}\left(x_{0}, y_{0}\right)$ denote the equilibrium point $P_{3}$ and its coordinates $\left(x_{0}, y_{0}\right)$. We define the Hopf bifurcation curve $\ell_{1}$ to be the curve in the parameter space onto which the equilibrium point $P_{3}\left(x_{0}, y_{0}\right)$ is critical and the divergence of system (9) equals $1-y_{0}+\nu=0$. Analogously, we define the zero-divergence curve $\ell_{2}$ to be the curve in the parameter space onto 
which the equilibrium point $P_{2}\left(x_{0}^{*}, y_{0}^{*}\right)$ renders the divergence of system (9) equal to $1-y_{0}^{*}+\nu=0$.

At the point $P_{2}\left(x_{0}^{*}, y_{0}^{*}\right)$, we have the curve $\ell_{2}: 1-y_{0}^{*}+\nu=0$. When $\mu \rightarrow 0, y_{0}^{*} \rightarrow 0$, so $\nu \rightarrow-1$. When $\frac{\mu}{\nu} \rightarrow-\frac{4}{27}, y_{*} \rightarrow \frac{1}{3}$, so $\nu \rightarrow-\frac{2}{3}, \mu \rightarrow \frac{8}{81}$. That is, the end points of $\ell_{2}$ are $B(-1,0)$ and $A\left(-\frac{2}{3}, \frac{8}{81}\right)$.

At the point $P_{3}\left(x_{0}, y_{0}\right)$, we have the Hopf bifurcation curve $\ell_{1}: 1-y_{0}+\nu=$ 0 . When $\mu \rightarrow 0, y_{0} \rightarrow 1$, and so $\nu \rightarrow 0$. When $\frac{\mu}{\nu} \rightarrow-\frac{4}{27}$, the double root of $\frac{4}{27} x^{3}+x+1=0$ is $x_{0}=-\frac{3}{2}$ and so $y_{0}=1+\frac{1}{x_{0}}=\frac{1}{3}$. Consequently, $\nu \rightarrow-\frac{2}{3}$ and $\mu \rightarrow \frac{8}{81}$. That is, the end points of $\ell_{1}$ are $(0,0)$ and $A\left(-\frac{2}{3}, \frac{8}{81}\right)$.

At every singular point $P\left(x_{0}, y_{0}\right)$, we have

$$
x_{0}-x_{0} y_{0}+1=0, \quad \mu x_{0}^{2}+\mu y_{0}=0 .
$$

From these two equations we get

$$
\frac{\mu+\nu y_{0}\left(y_{0}-1\right)^{2}}{\left(y_{0}-1\right)^{2}}=0 \quad \text { or } \quad \mu+\nu y_{0}\left(y_{0}-1\right)^{2}=0 .
$$

Consequently,

$$
\ell_{1}: \mu=-\nu^{3}(1+\nu)>0, \quad-\frac{2}{3}<\nu<0, \quad \lim _{\nu \rightarrow 0} \frac{d \mu}{d \nu}=0, \quad \lim _{\nu \rightarrow-\frac{2}{3}} \frac{d \mu}{d \nu}=\frac{4}{-27} .
$$

Similarly we have

$$
\begin{gathered}
\ell_{2}: \mu=-\nu^{3}(1+\nu)>0, \quad-1<\nu<-\frac{2}{3}, \\
\frac{d \mu}{d \nu}=-\nu^{2}(3+4 \nu) \begin{cases}<0, & -\frac{3}{4}<\nu<-\frac{2}{3}, \\
>0, & -1<\nu<-\frac{3}{4},\end{cases} \\
\lim _{\nu \rightarrow-1} \frac{d \mu}{d \nu}=1, \quad \lim _{\nu \rightarrow \frac{-2}{3}} \frac{d \mu}{d \nu}=\frac{-4}{27} .
\end{gathered}
$$

The maximum value of $\mu$ occurs at $\nu=-\frac{3}{4}$; it equals $\frac{27}{256}$. The curves $\ell_{1}$ and $\ell_{2}$ are depicted in Figure 2.

On $\ell_{1}$, the relative positions of the stable and unstable manifolds of the saddle point $P_{2}\left(x_{0}^{*}, y_{0}^{*}\right)$ can only be as depicted in Figure 4, case (b). This follows from the fact that on the Hopf bifurcation curve $\ell_{1}$ the quantity $\left.\operatorname{div}(9)\right|_{P_{2}}=1-y_{0}^{*}+\nu>0$ and also $P_{3}$ is unstable. Note that if cases (a) and (c) would occur, there would be at least one-sided stable periodic orbit surrounding $P_{3}$. However, that is impossible as was shown earlier in sub-section 3.4.

On the other hand, suppose $\left.\operatorname{div}(9)\right|_{P_{2}}=1-y_{0}^{*}+\nu \leq 0$. Observe that $y>y_{0}^{*}$ at every point on the whole stable and unstable manifolds of $P_{2}\left(x_{0}^{*}, y_{0}^{*}\right)$. Thus, $1-y+\nu<0$ and consequently, $\iint_{G}(1-y+\nu) d x d y<0$, where the region $G$ is bounded by $S=P_{2} N_{1} \cup \overline{N_{1} N_{2}} \cup N_{2} P_{2}$ as depicted in Figure 2 - case $7^{(1)}$.

$\overline{N_{1} N_{2}}$ is a segment on the outer-normal of $N_{2} P_{2}$ at Point $N_{2}$. By Green's Theorem [4]:

$$
\iint_{G}(1-y+\nu) d x d y=\oint_{S} X_{n}(x, y) d x=\int_{\overline{N_{1} N_{2}}} X_{n}(x, y) d s<0,
$$


where $X_{n}(x, y)$ denotes the projection of the vector field $((F(x, y), G(x, y))$ on the outer-normal of $S$. Thus the relative position of the stable and unstable manifolds of $P_{2}\left(x_{0}^{*}, y_{0}^{*}\right)$ must be as in Figure 4, case (a). By continuity, there must exist a saddle-loop line $\ell_{3}$ between $\ell_{1}$ and $\ell_{2}$.

One end point of $\ell_{3}$ is $A\left(-\frac{2}{3}, \frac{8}{81}\right)$ and the other is $C\left(\nu_{0}, 0\right)$ with $-1 \leq \nu_{0} \leq 0$. It is obvious that $\nu_{0} \neq-1$. If $\nu_{0}=0$, then when $\mu \rightarrow 0$ system (11) is transformed into

$$
\begin{aligned}
& \frac{d u}{d t^{\prime}}=-u z+u^{2}-u z^{2} \\
& \frac{d z}{d t^{\prime}}=u z-z^{2}-z^{3}
\end{aligned}
$$

The corresponding homogeneous system is

$$
\frac{d u}{d t^{\prime}}=u, \quad \frac{d z}{d t^{\prime}}=z
$$

Hence, as shown in Figure 3 on the Poincare sphere, the $\operatorname{singular}$ point $E(\tilde{E})$ is a critical node; i.e., along every ray there is a trajectory going into $E(\tilde{E})$. On the other hand, as $(\nu, \mu) \rightarrow\left(\nu_{0}, 0\right)$ along $\ell_{3}$, the saddle-loop becomes larger and larger. Thus, it will contradict the continuity of the vector field near $E$ and so $-1<\nu_{0}<0$.

In the region bounded by $\ell_{1}$ and the line $\frac{\mu}{\nu}=-\frac{4}{27}$, system (9) correspondingly has no saddle-loop. Suppose not; then there exists a saddle-loop $L^{*}$ for some $\left(\nu^{*}, \mu^{*}\right)$. Now $P_{3}$ is unstable and observe also that $L^{*}$ is repelling from the inside. Consequently, there is at least one stable limit cycle $L$ engulfed by $L^{*}$. In this region, $P_{3}$ is always unstable, so $L$ must have bifurcated from the saddle-loop $L^{*}$. From $\operatorname{div}(9)$ $\left.\right|_{P_{2}}=1-y_{0}^{*}+\nu>0$, we calculate the integral around $L^{*}$, namely,

$$
\oint_{L^{*}}\left(1-y+\nu^{*}\right) d t>0 .
$$

Consequently,

$$
\oint_{L}\left(1-y+\nu^{*}\right) d t>0
$$

whenever $L$ is sufficiently near $L^{*}$. That is, $L$ is unstable. Thus the contradiction proves the nonexistence of $L^{*}$.

By the arguments in sub-section 3.4, the saddle-loop and the limit cycle do not coexist. Hence, as one varies $(\mu, \nu)$ to consecutively traverse the regions (and curves) from $7^{(1)}, \ell_{1}, 7^{(2)}, \ell_{3}$, and $7^{(3)}$, we obtain the qualitative phase portraits as correspondingly depicted in Figure 2.

4.6. Case $8\left(\frac{\mu}{\nu}=-\frac{4}{27}\right.$ and $\left.\nu<0\right)$. The eigenfunction of system (15) is

$$
\lambda^{2}-\left(1-y_{0}+\nu\right) \lambda+\left(1-3 y_{0}\right) \nu=0 \text {. }
$$

On the line $\frac{\mu}{\nu}=-\frac{4}{27}$, we have $P_{2}\left(-\frac{2}{3}, \frac{1}{3}\right)$ and hence $\left(1-3 y_{0}\right) \nu=0$; i.e., one of the eigenvalues equals zero. When $\nu<-\frac{2}{3}$, respectively $\nu>-\frac{2}{3}$, the other eigenvalue is less than, respectively greater than, zero. So the singular point $P_{2}$ is a sink-saddle, respectively a source-saddle. At the point $A\left(-\frac{2}{3}, \frac{8}{81}\right)$, we have $\left(1-y_{0}+\nu\right)=0$ and 
$\left(1-3 y_{0}\right) \nu=0$. Hence both eigenvalues equal zero and the equilibrium point $P_{2}$ is degenerate.

Summary of the results. We first relate our system to a system in Chapter 7 of [10]. At the point $A\left(-\frac{2}{3}, \frac{8}{81}\right)$ in the parameter space $(\nu, \mu)$ we can transform the original system (9) about the singular point $x=-\frac{3}{2}, y=\frac{1}{3}$ to the system

$$
\begin{aligned}
& \frac{d x}{d t}=y+o\left(\epsilon^{2}\right), \\
& \frac{d y}{d t}=\nu_{1}+\epsilon \nu_{2} y+\epsilon x y+x^{2}+o\left(\epsilon^{2}\right),
\end{aligned}
$$

where $\nu_{1}, \nu_{2} \in o(1)$. System (26) is identically equation (7.3.12) in [10]. Thus, the results obtained for equation (7.3.12) in [10] also apply to system (9), near the singular point $\left(-\frac{3}{2}, \frac{1}{3}\right)$. Observe, however, that the results in [10] are only local. In contrast, the results in this paper are global.

We now summarize our results in the form of a theorem.

\section{Theorem.}

1. For system (9):

a) the parametric space $(\nu, \mu)$ is divided into eight regions by the curves: $\nu=0, \mu=0, \frac{\mu}{\nu}=-\frac{4}{27}$, the Hopf bifurcation curve $\ell_{1}: \mu=-\nu^{3}(1+\nu)$, $-\frac{2}{3}<\nu<0$, and the saddle connection curve $\ell_{3}$.

b) The end points of $\ell_{3}$ are $A\left(-\frac{2}{3}, \frac{8}{81}\right)$ and $C\left(\nu_{0}, 0\right)$, where $-1<\nu_{0}<0$.

c) $\ell_{1}$ and $\ell_{3}$ are tangent to the curve $\frac{\mu}{\nu}=-\frac{4}{27}$ at the point $A\left(-\frac{2}{3}, \frac{8}{81}\right)$.

2. Except for an analytic expression for the saddle connection curve $\ell_{3}$, the global bifurcation analysis of system (9) is completely determined.

3. System (9) has 13 different topological structures in the above regions and

\begin{tabular}{|c|c|c|}
\hline I. & $9,1^{(1)}$ & one focus, \\
\hline II. & $6^{(1)}, 6^{(2)}, 4$ & one saddle, \\
\hline III. & $5^{(1)}, 5^{(2)}$ & no singular points, \\
\hline IV. & $1^{(2)}$ & one degenerate node, \\
\hline V. & 2 & two saddle, one node, \\
\hline VI. & 3 & one saddle, one saddle-node, \\
\hline VII. & $8^{(1)}$ & one focus, one degenerate singular point \\
\hline VIII. & $8^{(2)}$ & one focus, one source-saddle, \\
\hline IX. & $8^{(3)}$ & one focus, one sink-saddle, \\
\hline $\mathrm{X}$. & $7^{(1)}$ on $\ell_{1}$ & two foci, one saddle, \\
\hline XI. & $7^{(2)}$ & $\begin{array}{l}\text { two foci, one saddle, one limit cycle } \\
\text { generated via Hopf bifurcation, }\end{array}$ \\
\hline II. & on $\ell_{3}$ & $\begin{array}{l}\text { saddle-connection, two foci, one saddle, } \\
\text { the limit cycle vanishes, the stable and } \\
\text { unstable manifolds of saddle point } P_{2} \\
\text { become coincident }\end{array}$ \\
\hline & $7^{(3)}$ & $\begin{array}{l}\text { two foci, one saddle } \\
\text { saddle connection vanishes. }\end{array}$ \\
\hline
\end{tabular}
boundaries, namely: 


\section{REFERENCES}

[1] F.M.A. Salam and S. Bai, Disturbance-generated bifurcations in a simple adaptive system: Simulation evidence, Systems Control Lett., (1986), 269-280.

[2] F.M.A. Salam, The sufficient richness and parameter convergence in MRAC systems: $A$ direct approach using the theory of Lyapunov, Proceedings of the 25th IEEE Conference on Decision and Control, (Deccember 1987), 13-15.

[3] Zhang Zhi-fen, About singular points of polynomial systems, preprint, (1986).

[4] G. Sansone and R. Conti, "Non-Linear Differential Equations," MacMillan, New York, 1964.

[5] A.A. Ahapohob, E.A. Neohtobny, N.N. Topaoh and A. Г. Manep, Kayectbehhai Teopni Hnhamuyecknx Cnctem btoporo nopinka, nza. Hayka, (1966), 241-249, 3770410.

[6] Zhang Zhi-fen, Ding Tongren, Huang Wanzao and Dong Zhenxi, "Qualitative Theory of Ordinary Differential Equations," Press of Science, Peking (1985).

[7] Li Chengzhi, Two problems of planar quadratic systems, Scientia Sinica (series A), 26 (1983), 471-471.

[8] F.M.A. Salam, Parameter space analysis and design of an adaptive system, Proceedings of the 25th IEEE Conference on Decision and Control, (December 1986), 1155-1160.

[9] Zhang Zhi-fen, Proof of the uniqueness theorem of limit cycles of generalized Lienard equations, Applicable Anal., 23 (1986), 63-76.

[10] J. Guckenheimer and P.J. Holmes, "Nonlinear Oscillation, Dynamical Systems and Bifurcations of Vector Fields," Applied Math. Sci., 42, Springer, Berlin, New York, 1983.

[11] F.M.A. Salam, S.A. Van Gils and Z. Zhi-fen, Global bifurcation analysis of an adaptive system, The Proceedings of the 27th IEEE Conference on Decision and Control, December 1988.

[12] F.M.A. Salam, The Melnikov technique for highly dissipative systems, SIAM J. Appl. Math., 47 (1987), 232-243.

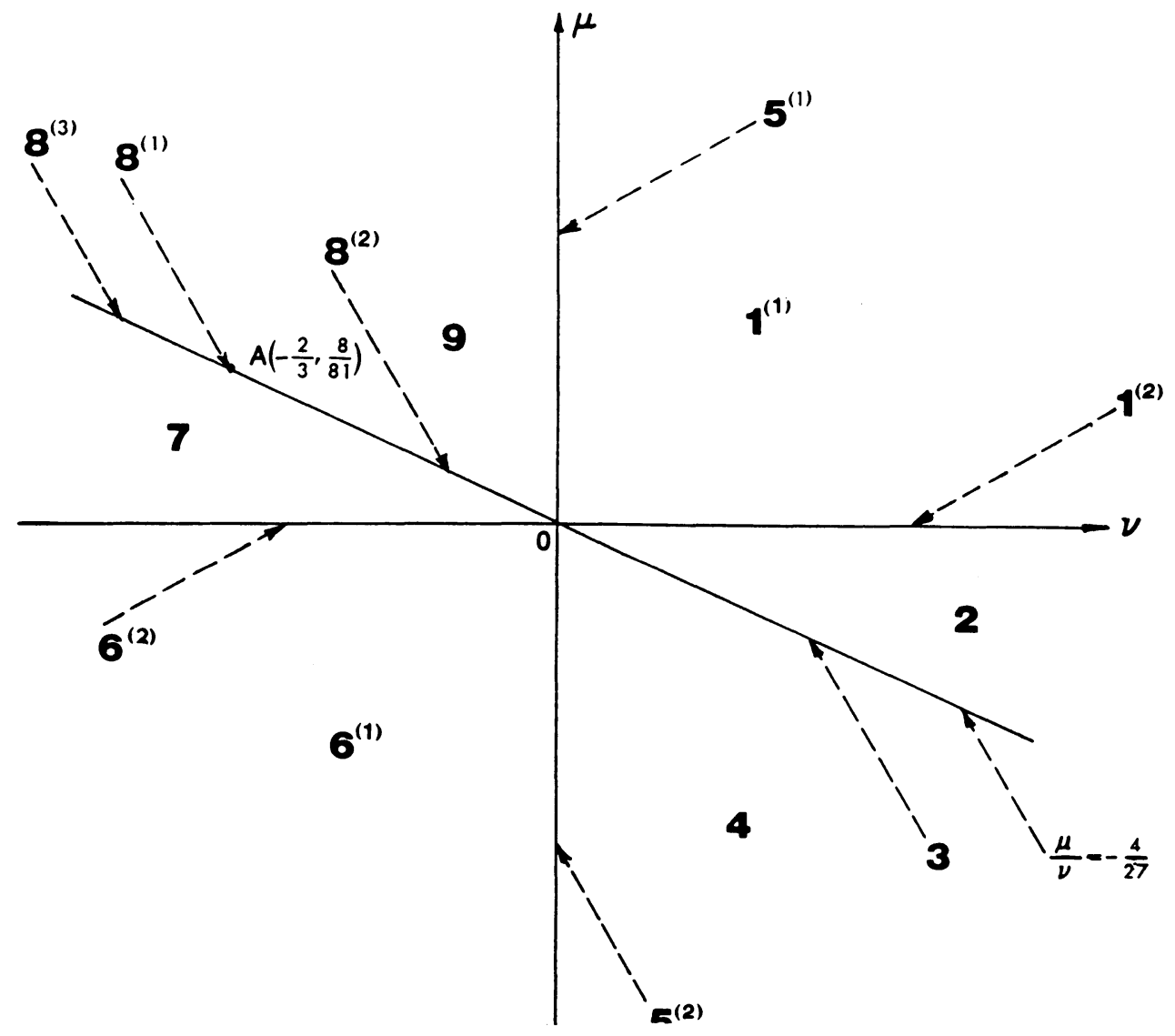




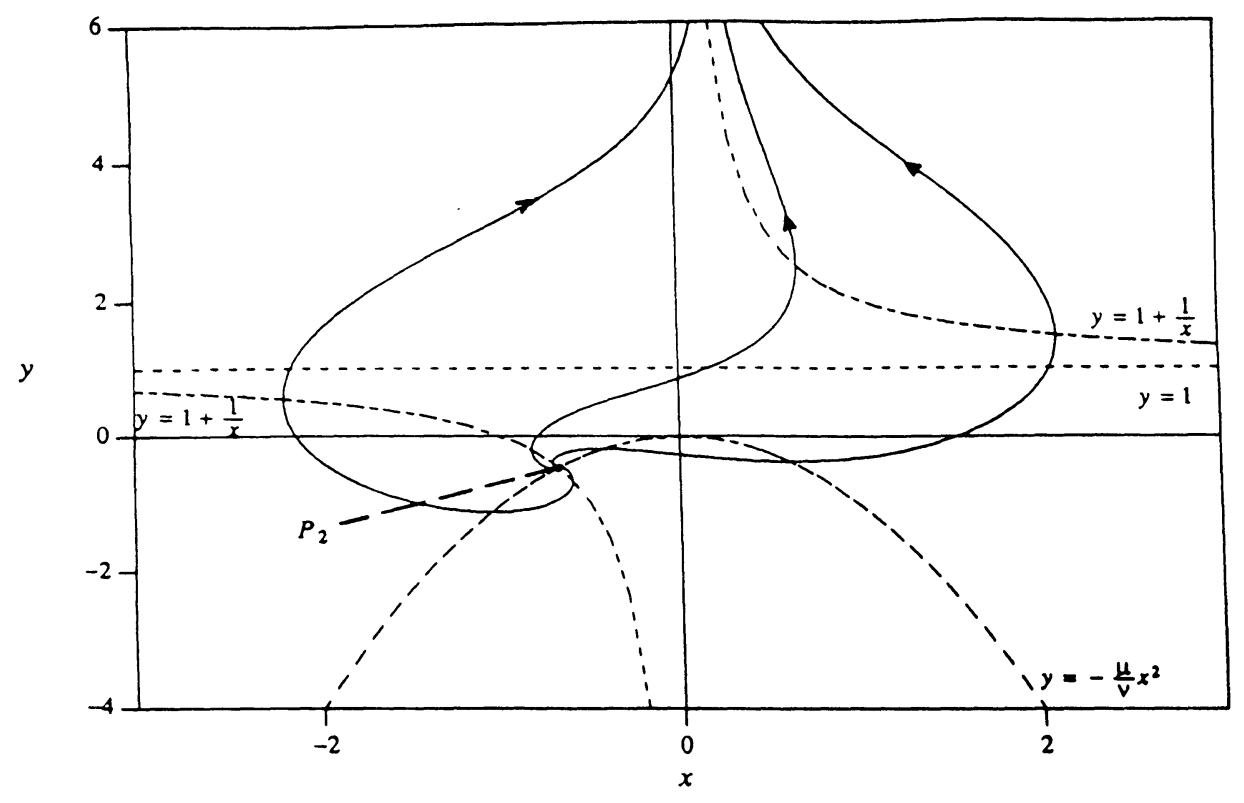

I(1): $P_{2}$-focus, $v>0, \frac{\mu}{v}>0$

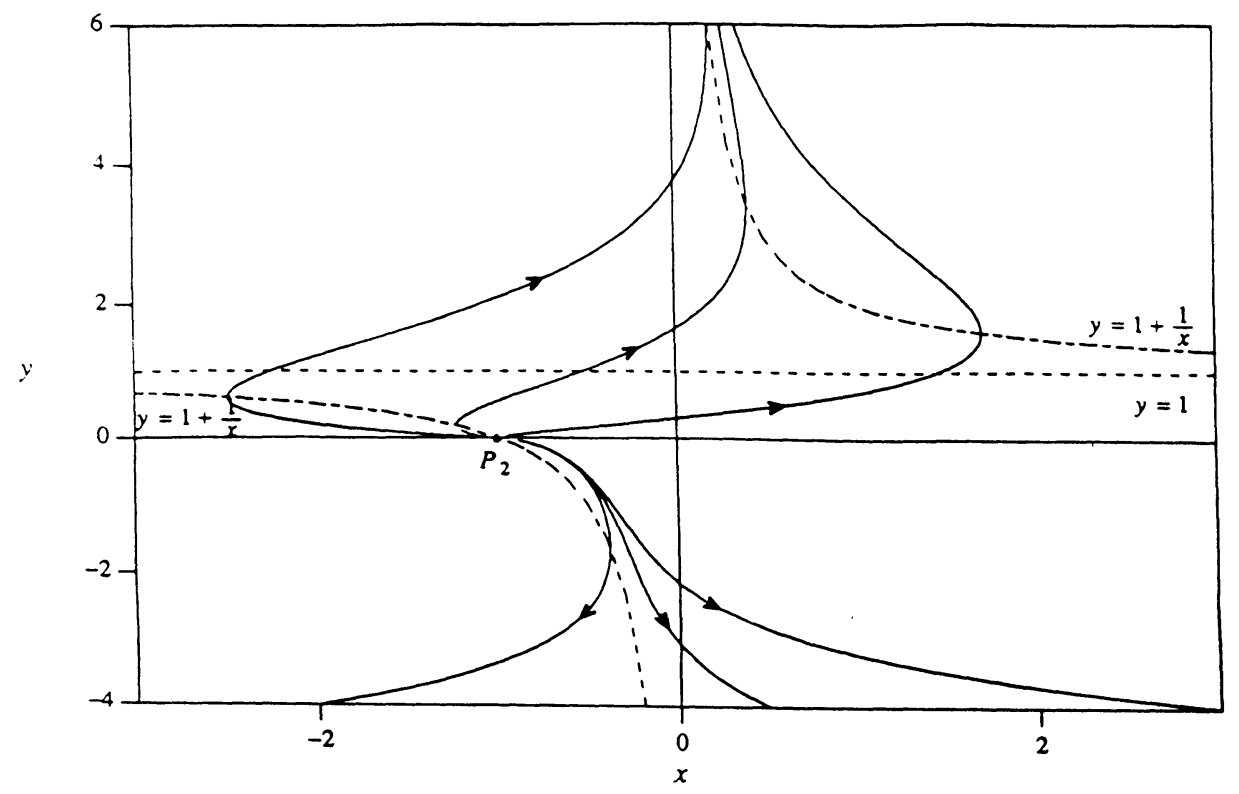

1(2): $P_{2}$-node $(v \neq 1)$, degenerate node $(v=1), v>0, \mu=0$ 


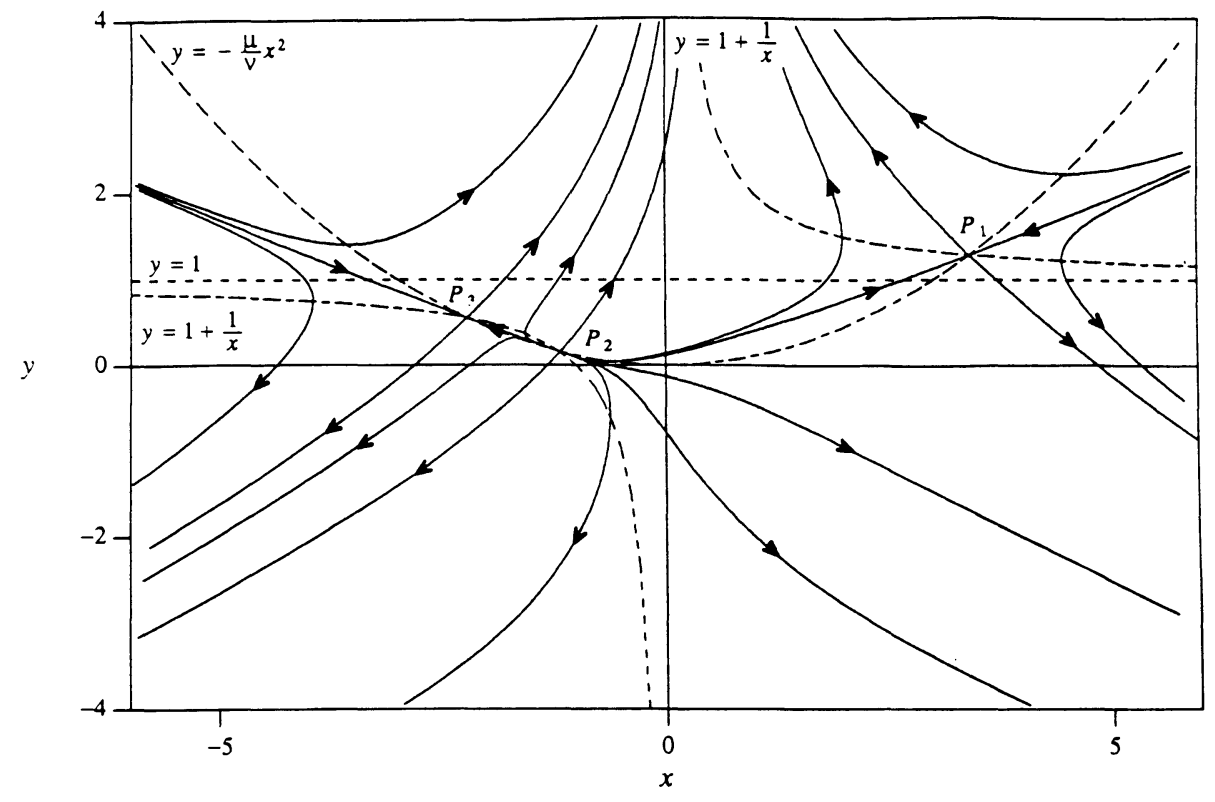

2: $P_{2}$-node, $P_{1}, P_{3}$-saddle,

$v>0,-\frac{4}{27}<\frac{\mu}{v}<0$

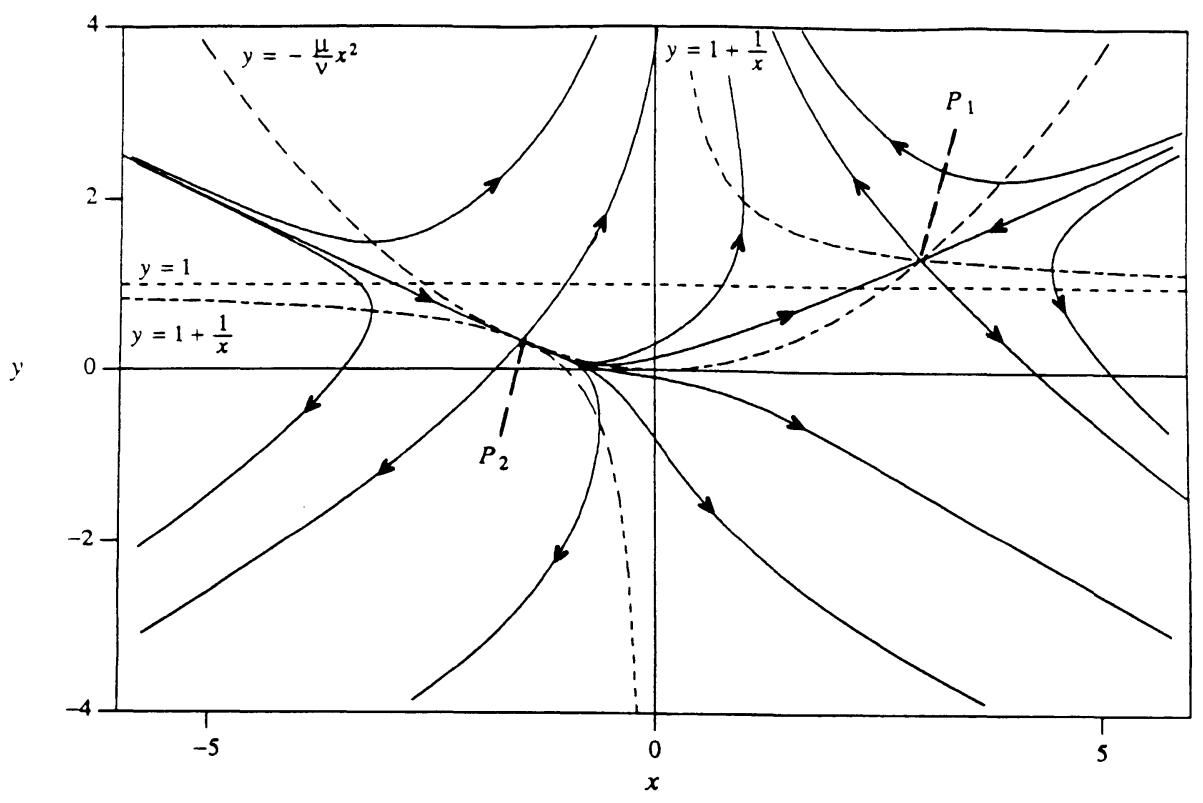

3: $P_{1}$-saddle, $P_{2}$-saddle node,

$$
v>0,-\frac{4}{27}=\frac{\mu}{v}
$$




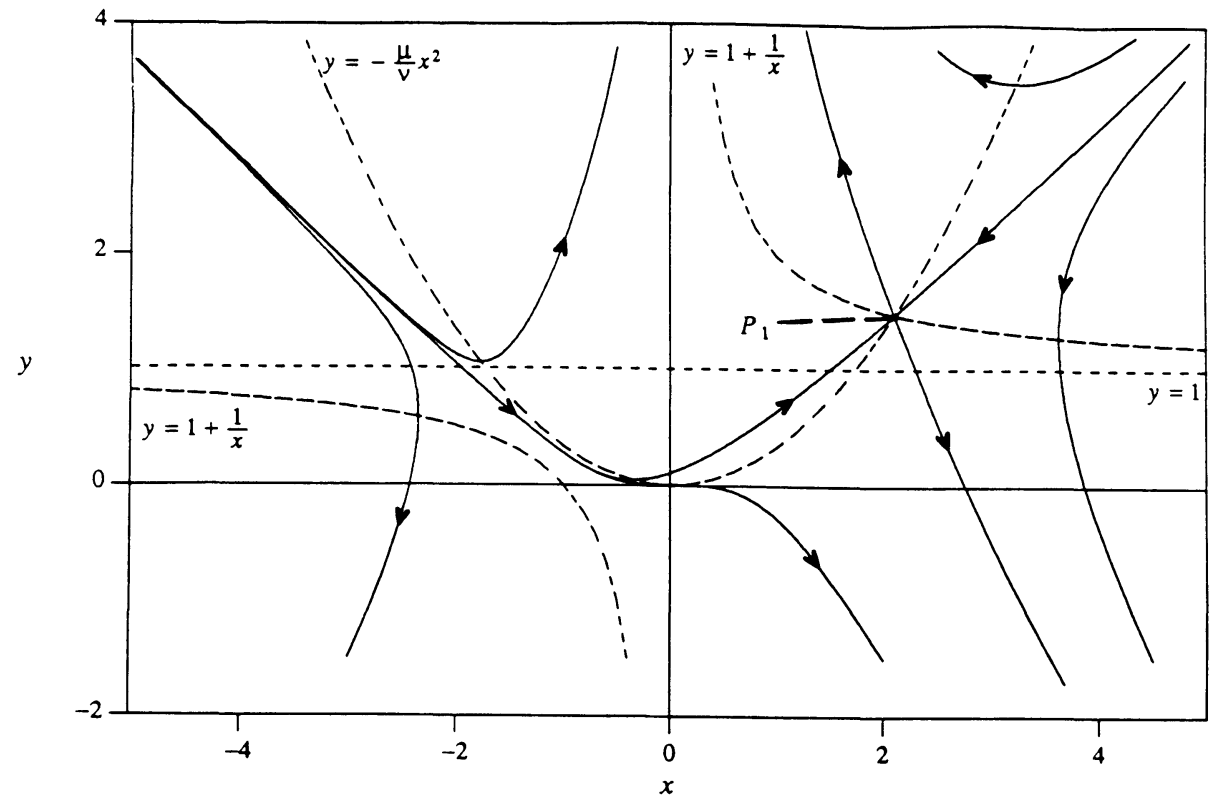

4: $P_{1}$-saddle,

$v>0,-\frac{4}{27}>\frac{\mu}{v}$

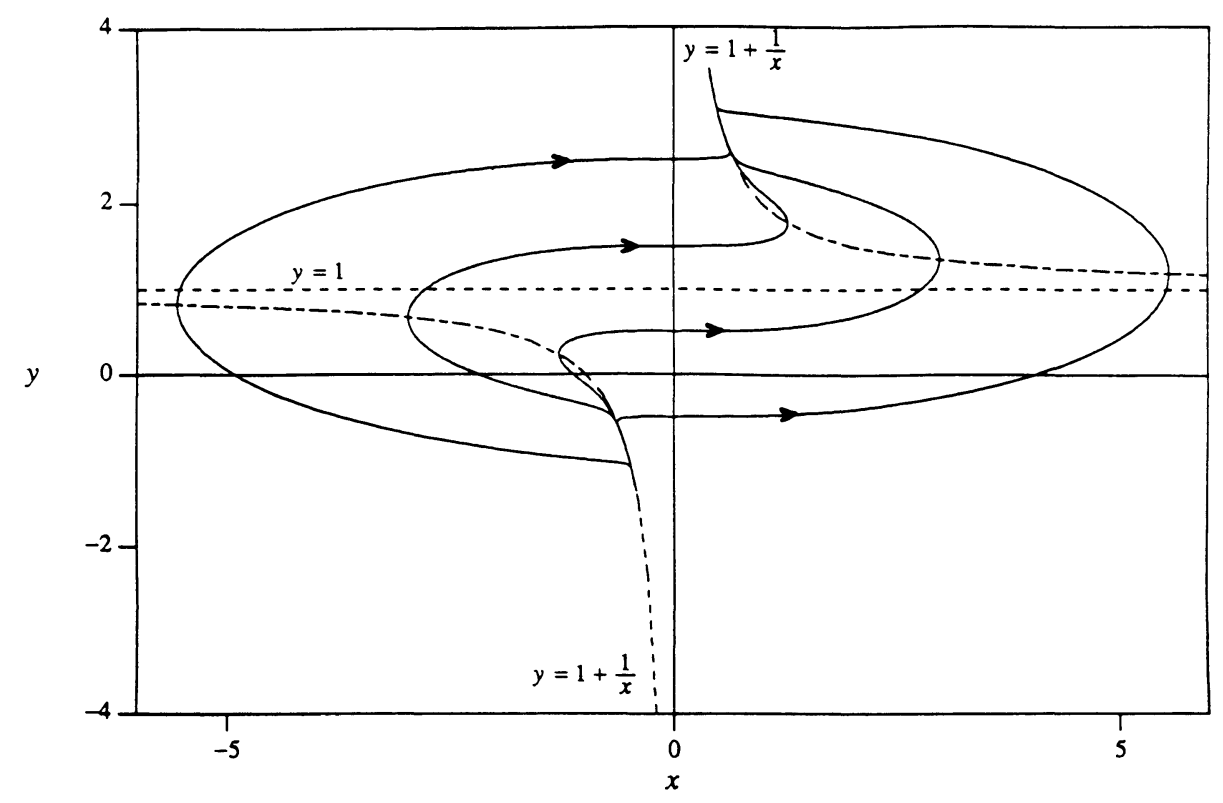

$5^{(1)}: \mu>0, v=0$ 


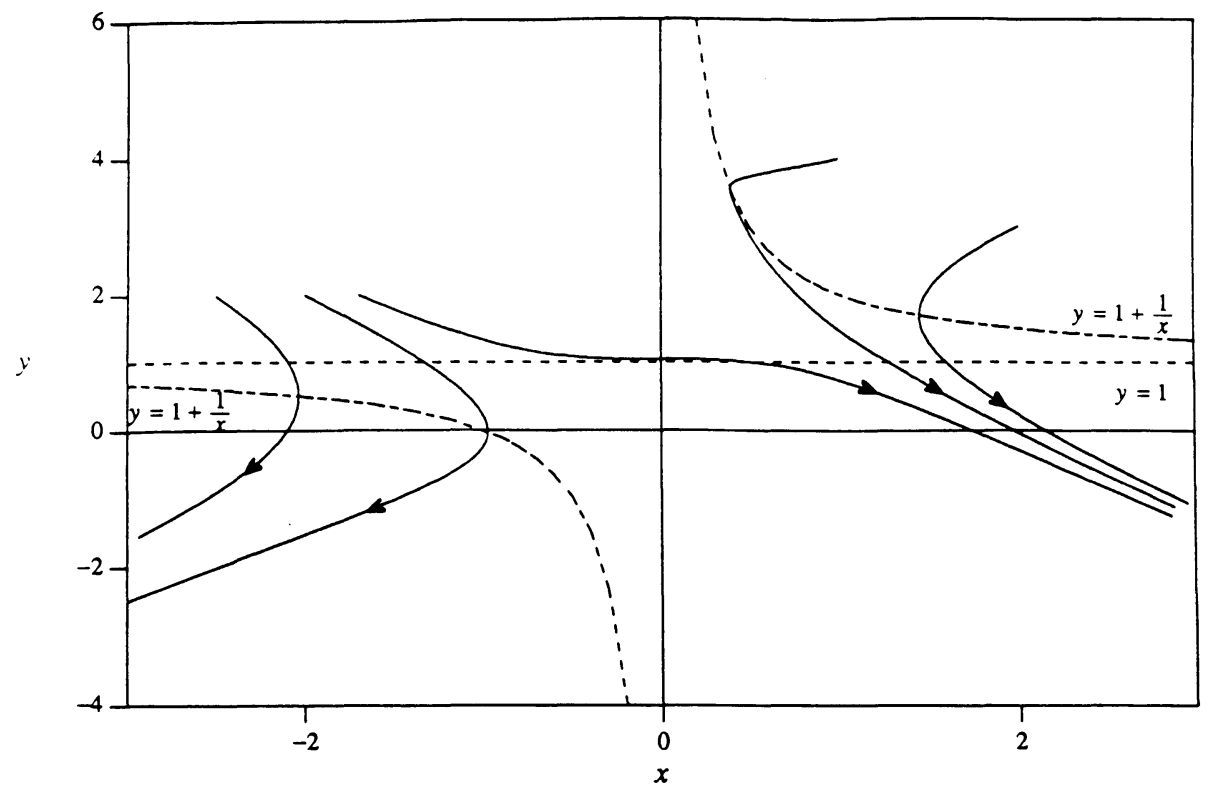

5(2): $v=0, \mu<0$

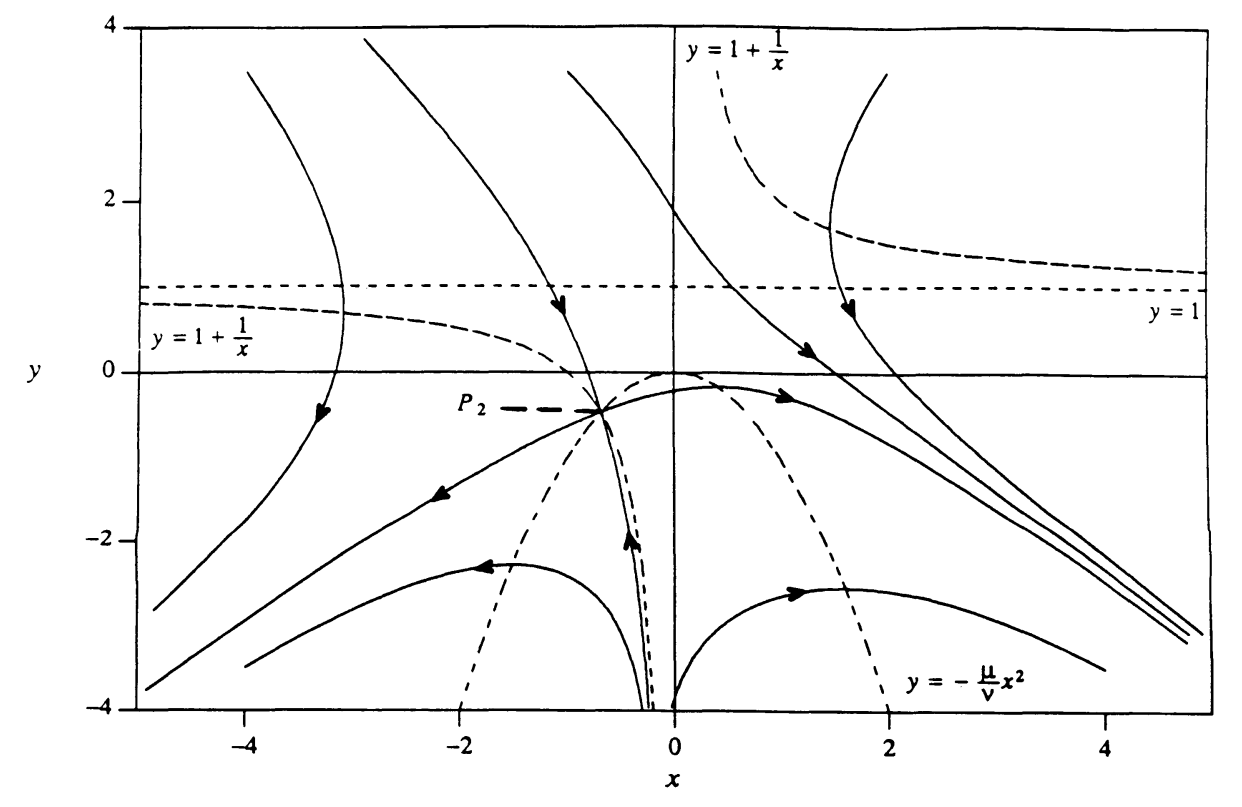

$6^{(1)}: P_{2}$-saddle,

$v<0, \frac{\mu}{v}>0$ 


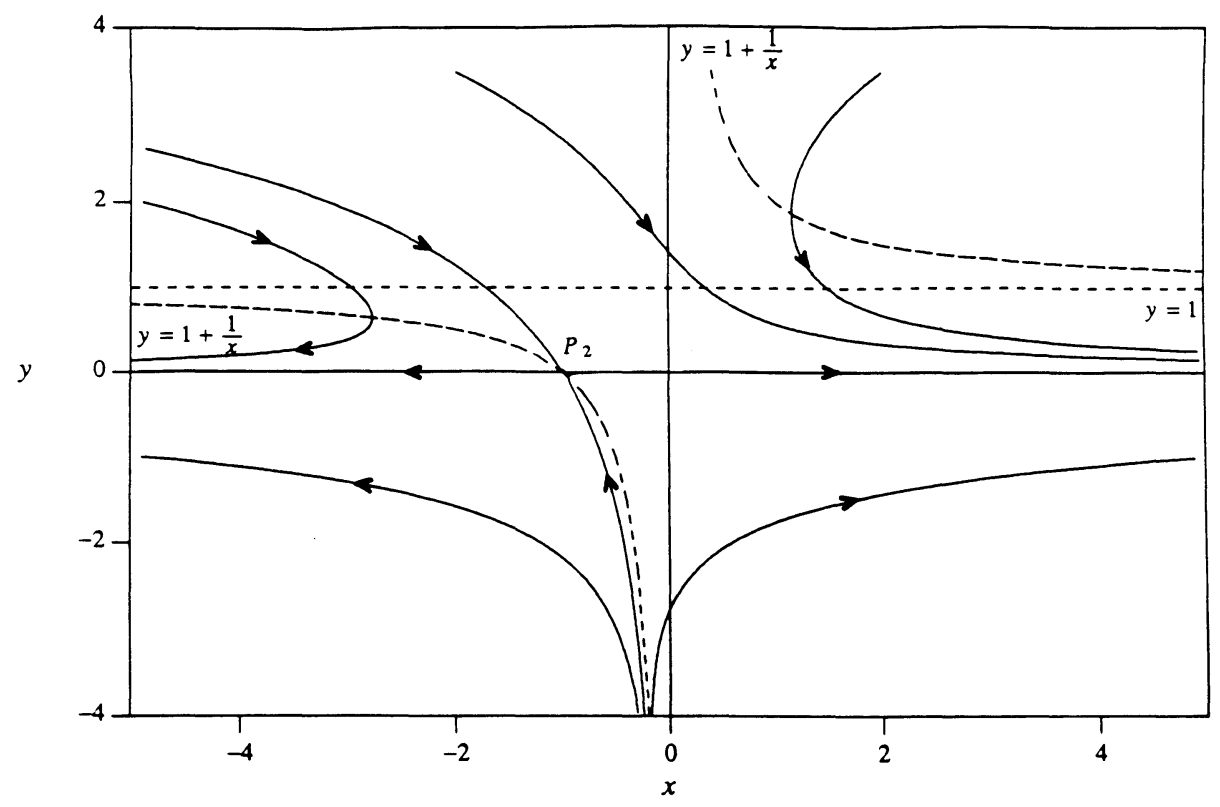

6(2) : $P_{2}$-saddle,

$v<0, \mu=0$

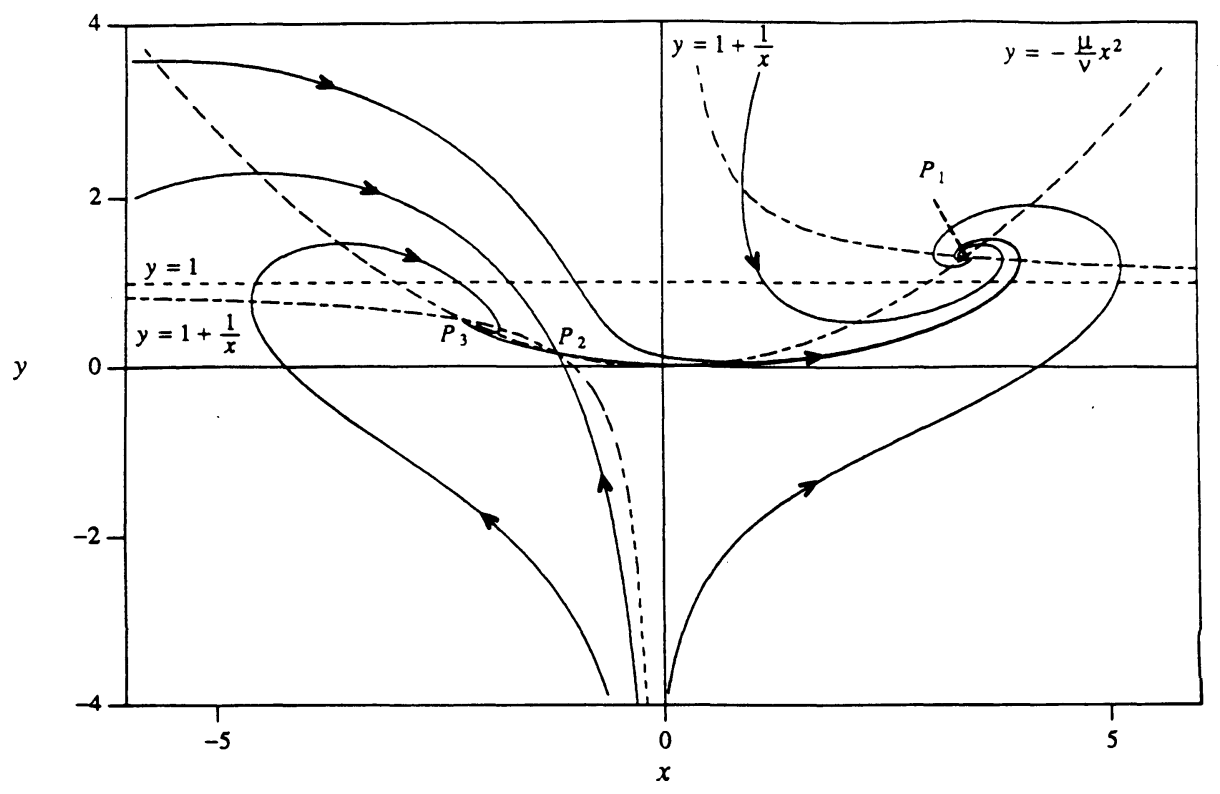

7: $P_{2}$-saddle, $P_{1}, P_{3}$-focus,

$v<0,-\frac{4}{27}<\frac{\mu}{v}$ 


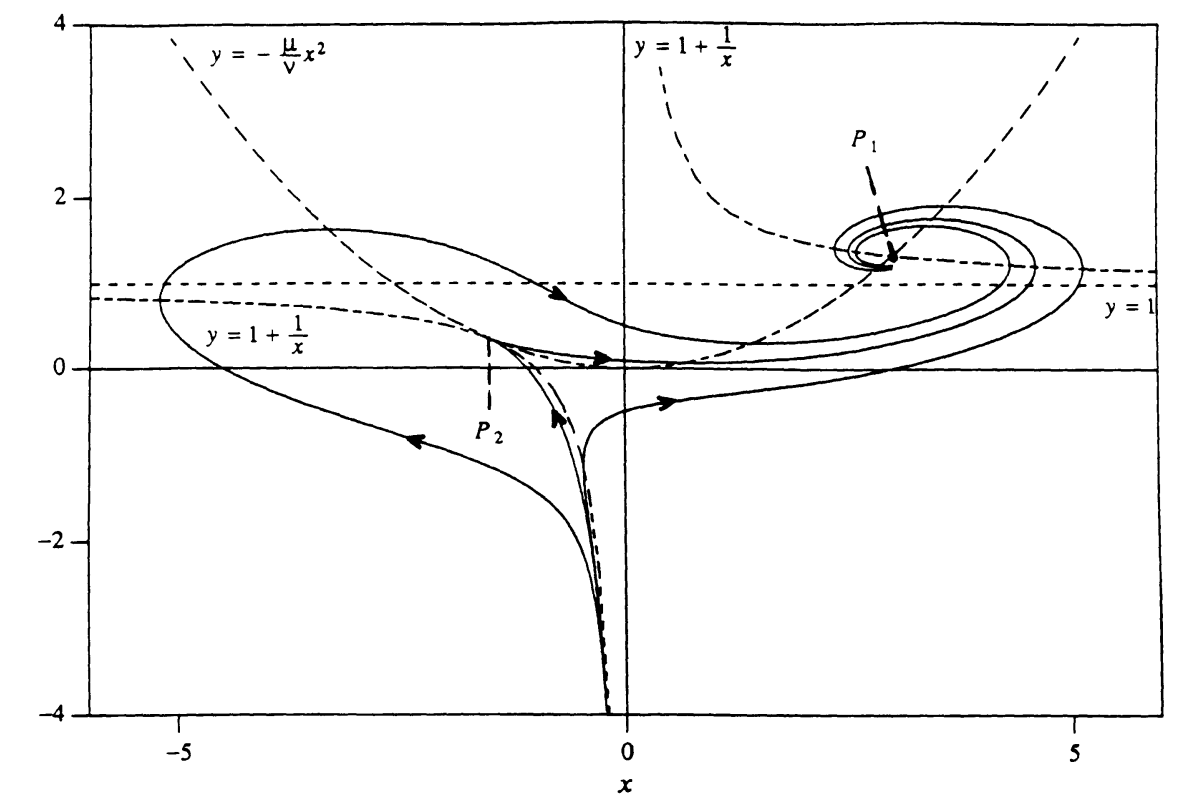

8(1): $P_{1}$-focus, $P_{2}$-degenerate singular point,

$$
v=-\frac{2}{3}, \frac{\mu}{v}=-\frac{4}{27}
$$

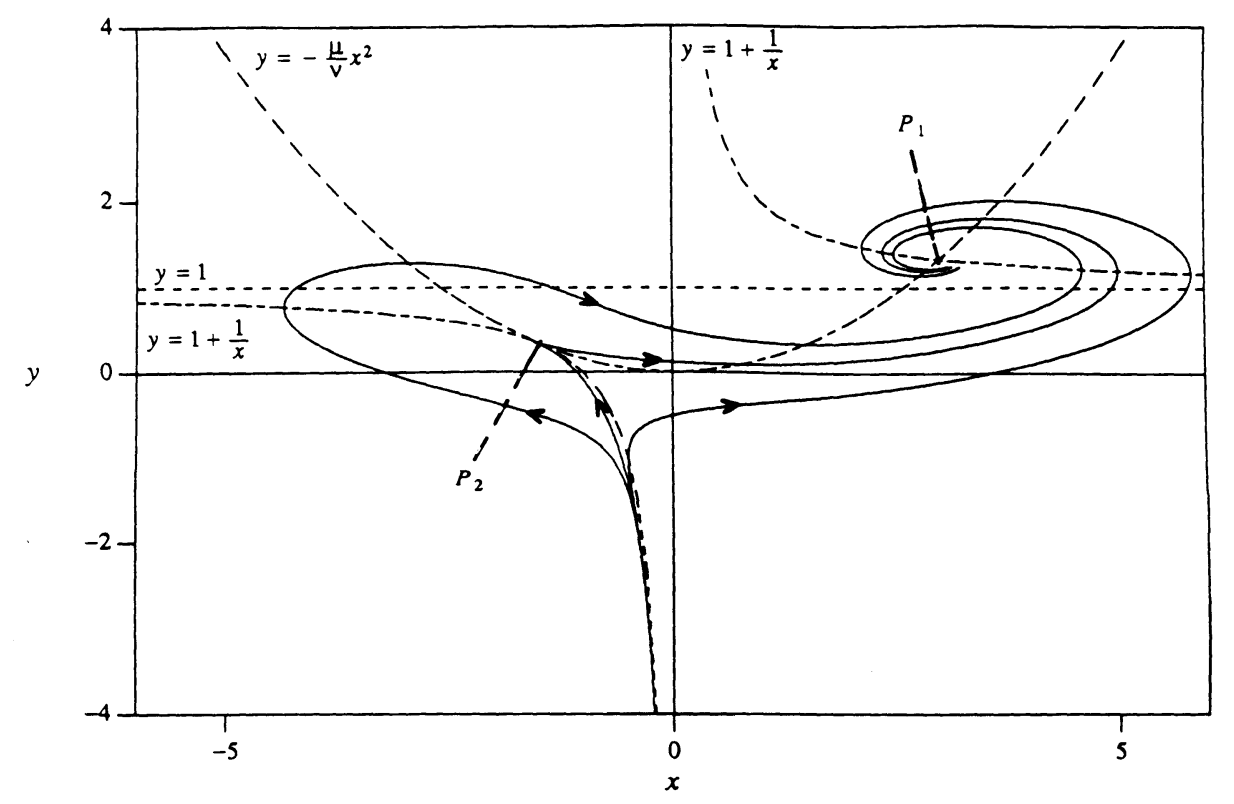

8(2): $P_{1}$-focus, $P_{2}$-source-saddle,

$$
-\frac{2}{3}<v<0, \frac{\mu}{v}=-\frac{4}{27}
$$




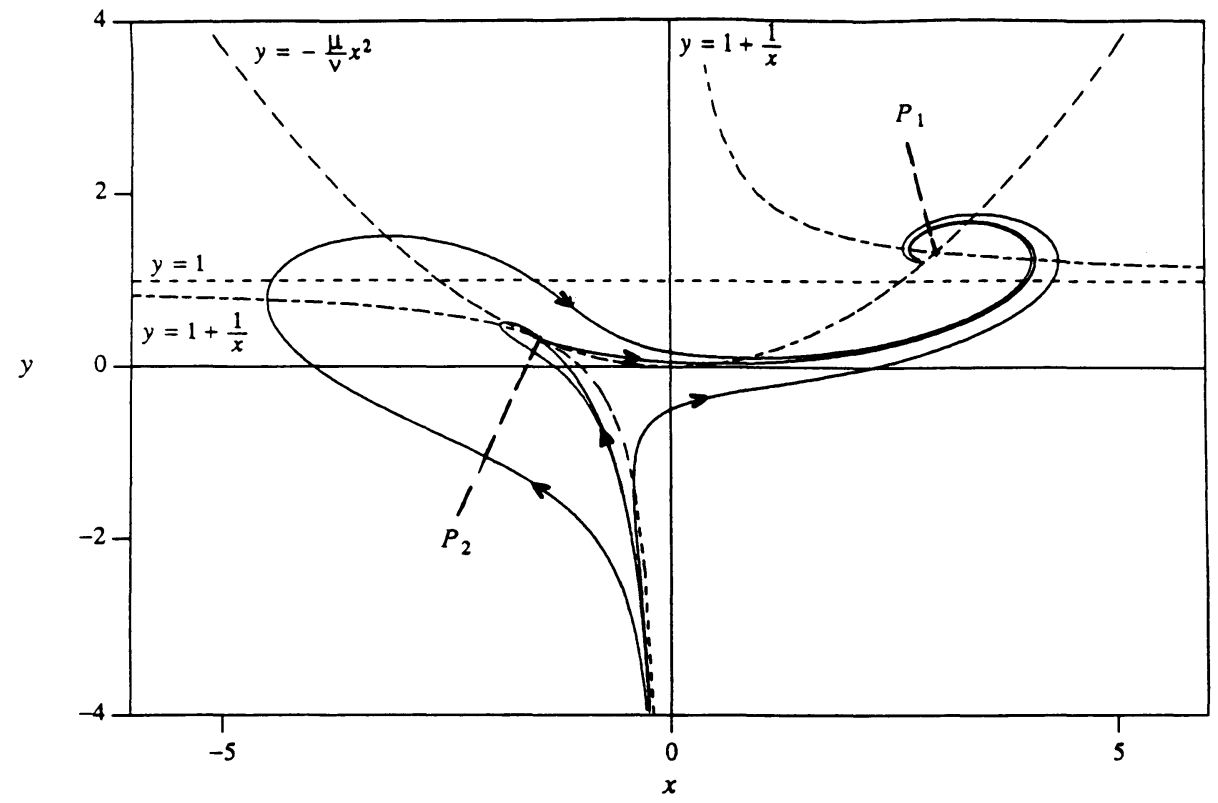

8(3): $P_{1}$-focus, $P_{2}$-sink-saddle,

$$
v<-\frac{2}{3}, \frac{\mu}{v}=-\frac{4}{27}
$$

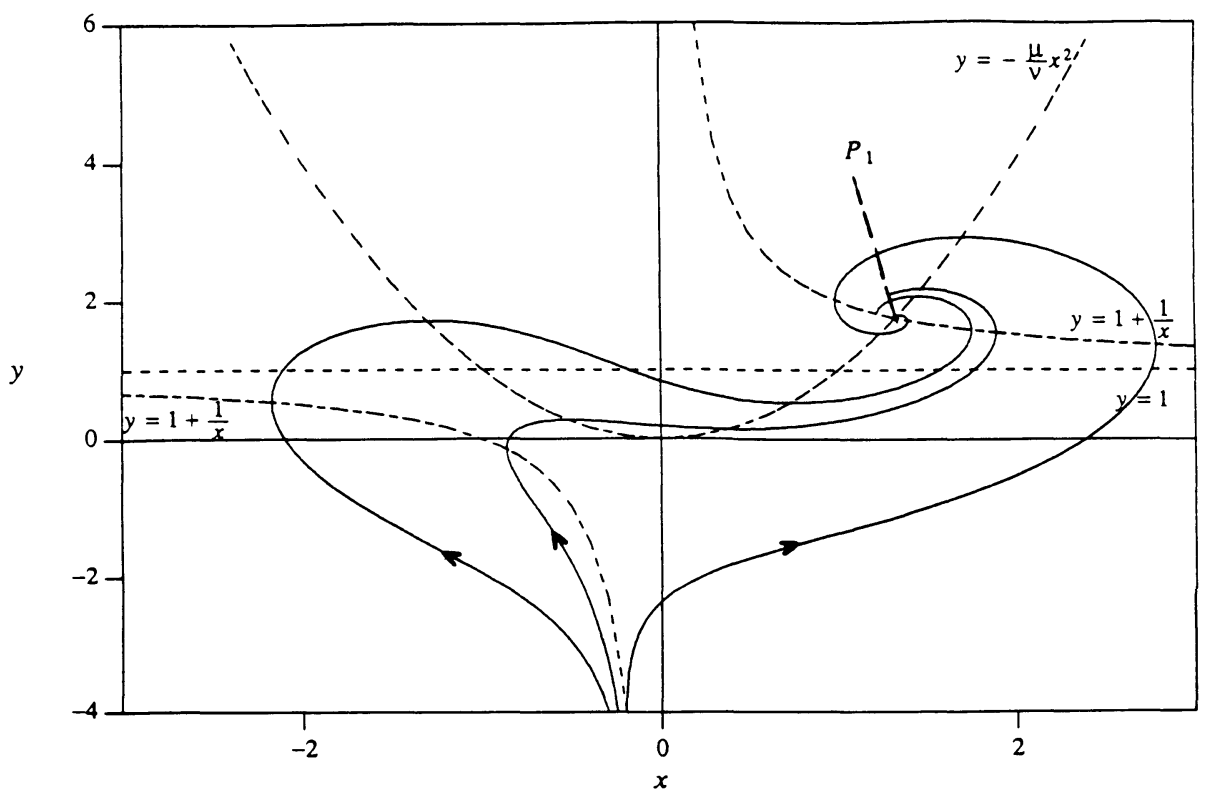

9: $P_{1}$-focus, $v<0, \mu \leq-\frac{4}{27}$ 


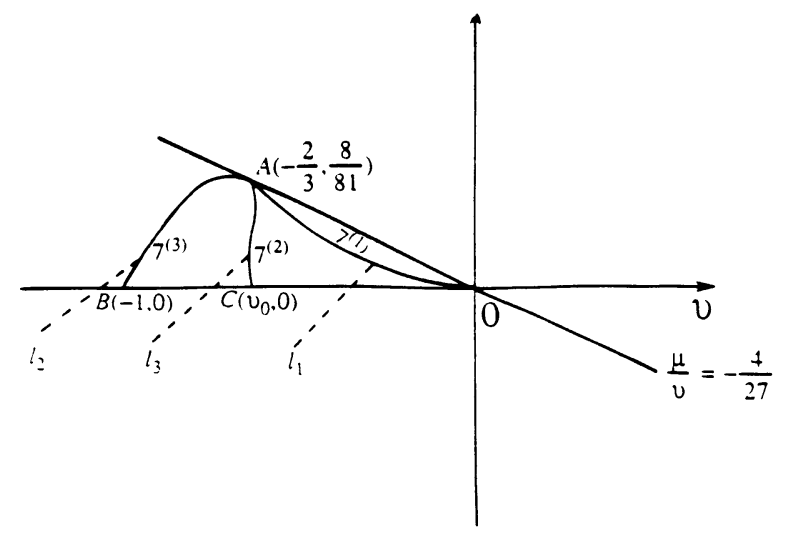

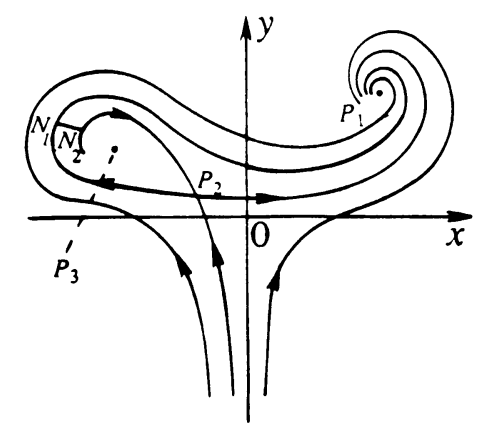

$7^{(1)}$ and $l_{1}$

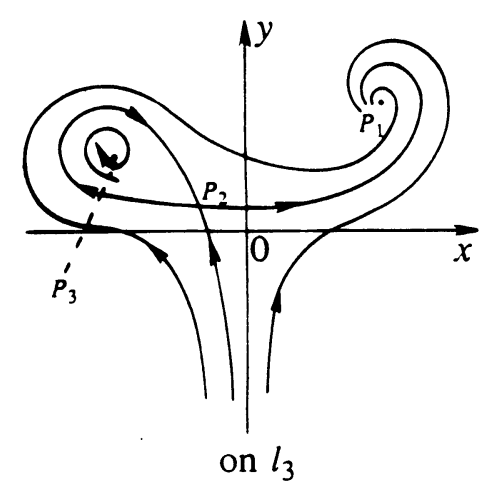

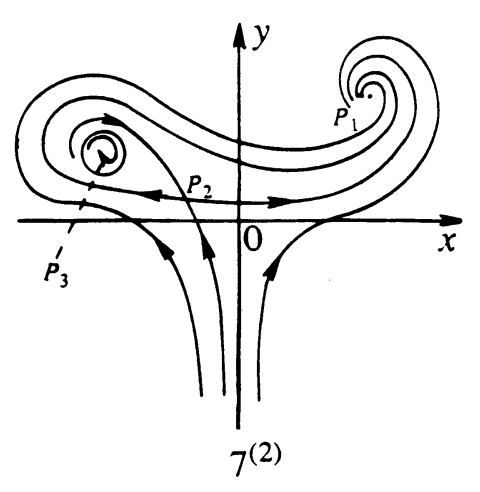

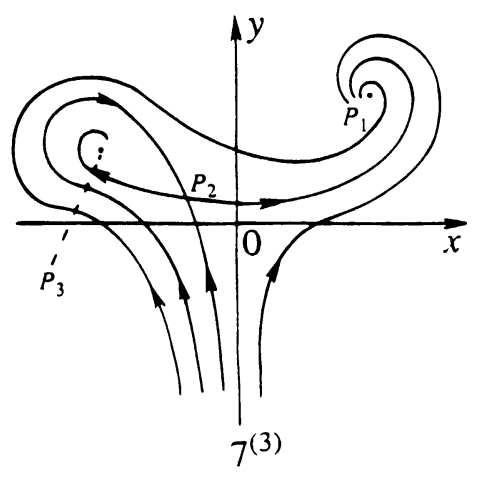

Figure 2 


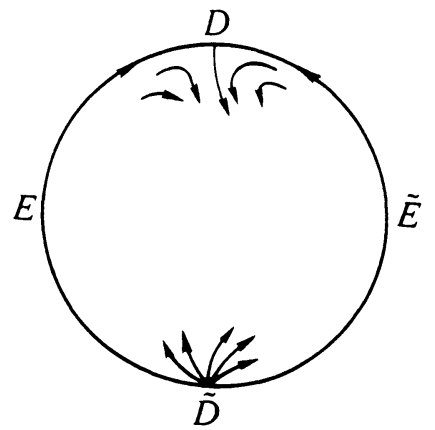

Figure 3

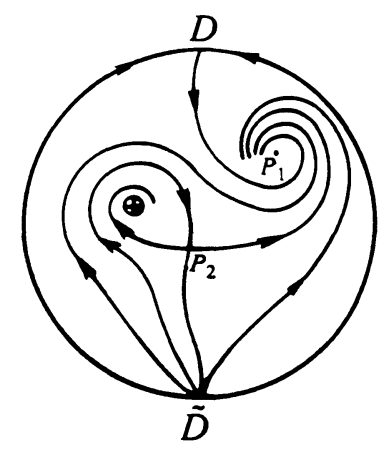

$\oplus$ - attractor

(a)

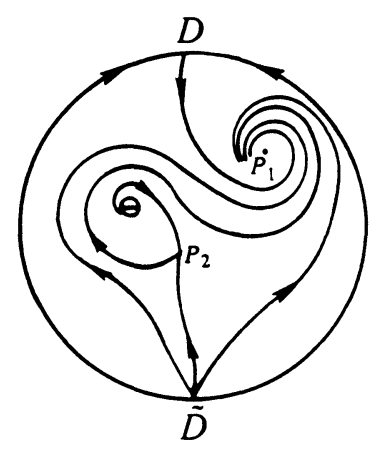

$\Theta$ - repeller

(b)

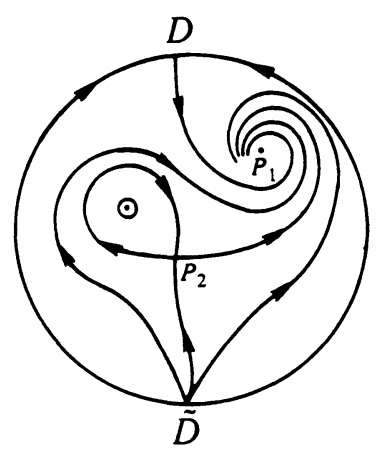

$\odot$ - attractor or repeller

(c)

Figure 4 